\title{
Integration Methods of Cyclodextrins on Gold and Carbon Electrodes for Electrochemical Sensors
}

\author{
Maria Antonietta Casulli ${ }^{1, *}$, Irene Taurino ${ }^{2}$, Sandro Carrara ${ }^{3}$ and Takashi Hayashita ${ }^{1}$ (D) \\ 1 Lab of Analytical Chemistry, Department of Materials and Life Sciences, Faculty of Science and Technology, \\ Sophia University, Tokyo 102-8554, Japan; ta-hayas@sophia.ac.jp \\ 2 Integrated Systems Laboratory, Ecole polytechnique fédérale de Lausanne (EPFL), 1015 Lausanne, \\ Switzerland; Irene.Taurino@pmi.com \\ 3 Integrated Circuits Laboratory, Ecole polytechnique fédérale de Lausanne (EPFL), 1015 Lausanne, \\ Switzerland; sandro.carrara@epfl.ch \\ * Correspondence: m-casulli-5h9@eagle.sophia.ac.jp
}

Received: 14 October 2019; Accepted: 25 November 2019; Published: 27 November 2019

\begin{abstract}
Cyclodextrins (CDs) are oligosaccharides composed of six $(\alpha)$, seven $(\beta)$ or eight $(\gamma)$ glucose units. Their inner hydrophobic cavity and hydrophilic external surface enable the formation of the "host-guest inclusion complex" with different organic or inorganic molecules showing high molecular selectivity. For these characteristics, CDs have many potential applications in electrochemical sensing. To enable CDs immobilization on the electrode surfaces, different chemical modifications are needed depending of the electrode material, while nanomaterials have been exploited to enhance the sensing signal. The CDs binding onto gold nanoparticles or carbon nanotubes, as an electron-transfer mediator to the electrode surface, is a typical example of it, while also graphene is largely used. The aim of the present review is to give an overview of CDs properties and their applications to electrochemical sensors for medical diagnostics. Different kinds for the functionalization of CDs onto electrode surfaces will be reviewed as well as their performance in presence of nanomaterials. Finally, CDs-based devices for sensing biomedical molecules of biomedical interest will be briefly presented and discussed.
\end{abstract}

Keywords: cyclodextrins; gold electrode; carbon electrode; functionalization; electrochemical sensor; biomedical sensors

\section{Introduction}

"Personalized health care" is an urgent need that is taking more and more relevance in medicine. It consists in calibrate and regulate decisions, practices and interventions, depending on the individual patient' characteristics and includes many fields of medicine, from diagnostic to therapy. Particularly, therapy is one of the major branches of medicine that may benefit of "personalized treatments" since it approaches the right doses at the right moment, avoiding to expose the patient to un-intentional risks. Indeed, drug response is different for every patient because it depends on genetics, epigenetics and phenotyping, while many other factors (e.g., attitude of life, age, environments, environmental exposure, etc.) take a role as well. For these reasons, monitoring human metabolism in real time would be more often required to obtain "the right drug, at the right dose, in the right moment" to finely adjust the drugs therapy on each single patient.

Over the years, electrochemical devices have been proposed for the detection of organic and biomolecules due to their easy and low-cost manufacturing processes as well as for the possibility to realize a multi-panel system for the simultaneous detection of several human metabolites, also "in continua" instead of one-shot measurements [1]. Moreover, they also allow easy assembly with electronic systems for reading and transmitting acquired data. 
On the other hand, electrochemistry is in continuous progress: enzyme-based biosensors have been largely developed for sensing metabolites like glucose and lactate, but also for the detection of drugs (e.g., cytochromes-based biosensors proposed for the detection of etoposide, ifosfamide, cyclophosphamide and ftorafur [2], four well known used anti-cancer compounds largely used in chemotherapy).

However, enzymes possess some intrinsic limitations, such as the lowering of their activity in time once attached onto electrode surfaces [3], which may also result in decreasing-in-time the performance of enzyme-based biosensors as, for example, registered in the continuous monitoring of the naproxen [4] a well-known anti-inflammatory compound.

For these reasons, new sensing strategies have been considered and cyclodextrins (CDs) have been selected as potential candidates to provide selectivity to future electrochemical sensing-devices. Cyclodextrins are oligosaccharides composed of six $(\alpha)$, seven $(\beta)$ or eight $(\gamma)$ glucose units. Their inner hydrophobic cavity and hydrophilic exterior are enabled to form a "host-guest inclusion complex" targeting different organic or inorganic molecules, toward which usually show high molecular selectivity.

Particularly for electrochemical devices, $\beta$-CDs are the most commonly used since their ring-size well matches with a large number of target diagnostic-molecules and since, usually, they are enough low-costs material [5].

Therefore, the aim of the present review is to provide an overview of CDs properties and of the different methods of integration in electrochemical sensors. Various chemical modifications of CDs will be described as well, and the possibility of their co-immobilization with nanomaterials for enhanced sensing will be described too. The final section closes the paper by focusing on CDs-based sensors for biomedical applications.

\section{Chemical Modifications of CDs for Electrochemical Devices}

The hydroxy groups at the edge of inner cavity of the cyclodextrins establish hydrogen bonds among their $\mathrm{OH}$ groups to form their classic bucket structure and provide electrostatic and dipole-dipole interactions with target molecules. In fact, depending on the stereoscopic structure and on the matching-size of the target-molecule, typically the "host-guest inclusion complexes" with CDs is resulting in a specific binding-constant and binding-strength. Considering the hydrophilic nature of the external walls of the cavity, the main forces for the molecular recognition are indeed provided mostly by hydrogen bonds in natural CDs or mostly by electrostatic ones in CDs chemically modified.

Among the several kinds of cyclodextrins, $\beta$-CDs are the most commonly used in electrochemistry since their cavities allow the best-performing inclusion. However, they are the less soluble in water than $\alpha$-CDs and $\gamma$-CDs, since their intermolecular hydrogen bonds prevent the formation of hydrogen bonds with the surrounding water molecules [5].

The solubility in water of CDs increases by introducing chemical functional groups in the surface of the molecule. This usually also results in better capacity of immobilization onto substrates for sensing aims.

Starting from natural CDs, it is possible to synthetize different kinds of CDs derivatives [5].

The first type of CDs derivatives belong to the class of carboxymethyl-CDs (CM-CDs) [6]. In this class, the carboxymethyl group located at the edge of the cavity changes the characteristic hydrogen bond configuration of CDs since hydrogen bonds are established among the hydrogen atoms of the carboxyl groups and the hydrogen atoms of the water molecules, which improve the CDs solubility in water $[5,7]$.

Instead of in hydroxyl groups, the second type of CDs derivatives is obtained by the introduction of amino-groups, typically with an ethylenediamine on the 6th position of the $\mathrm{CD}$. The amino-groups at the edge of the cavity establish hydrogen bonds and dipolar interactions with other water molecules. On the other hand, under acidic conditions, amino groups go to protonation that results in electrostatic interactions and hydrogen bonds with other interacting molecules [5]. 
Since they can form a covalent bonding with gold electrode or gold nanoparticles [8,9] (GNPs), a third category of CDs of interest in electrochemistry is that of thiolated-CDs (SH- $\beta$-CDs) $[10,11]$ (both mono or multi-thiolated). Weisser et al. [12] investigated four different kinds of mono or multi $\mathrm{SH}-\beta$-CDs with different chain-length, with respect to their absorption and orientation onto gold electrodes. They concluded that the mono-thiolated CDs with a long carbon chain can form densely packed monolayers but also physisorbed undesired adlayers. Another approach is to obtain SH-CDs by reduction with mercaptoethanol after the substitution of tosylated-CDs (TS-CDs). In this way, multi-thiolated CDs with amino-group inserted in the chain have been obtained [13-15].

Another possible CDs derivative class is that of hydroxypropyl-CDs (HP-CDs), instead largely used on carbon substrates. The most common method to synthetize HP-CDs is through a reaction of condensation of $\beta$-CDs with propylene oxide, at low temperature and under catalysis in basic solution $(\mathrm{NaOH})[5,16]$.

Other CDs derivatives for more specific electrochemical applications are represented by succinyl-CDs [17], per-6-deoxy-per-6-(2-carboxy-methyl)thio- $\beta$-cyclodextrin (AcSCD) [18], lipoyl-CDs [19] or disulfides bridged two adjacent $\beta$-CDs (DB- $\beta$-CDs) [20].

Possible chemical modifications of $\mathrm{CDs}$ and their interactions with different electrode materials are shown in Table 1.

Table 1. Cyclodextrins (CDs) chemical modifications and their kind of functionalization to respective substrates.

\begin{tabular}{ccc}
\hline CD Derivative & Kind of Interaction & CDs Integration Substrate \\
\hline CM-CDs & electrostatic interaction amide linkage & gold \\
NH-CDs & electrostatic interaction amide linkage & gold/carbon \\
SH-CDs & Au-SH linkage & gold \\
HP-CDs & electrostatic interaction & carbon \\
\hline
\end{tabular}

\section{Immobilization Strategies on Electrode Surfaces}

As mentioned before, nanomaterials are often used to functionalize metallic surfaces for sensing aims. In literature, there are many methods for binding CDs to nanomaterials such as gold nanoparticles (GNPs) [21], or carbon nanomaterials such as carbon nanotubes (CNTs) [22], graphene [23] or fullerenes [24]. On the other hand, literature also shows many examples of inclusion of CDs in conductive polymers [25] to functionalization aims. Bottom up techniques have also been proposed [26] as a possibility to deposit CDs directly onto the electrode surface. In the following three paragraphs different kinds of CDs immobilization onto gold and carbon electrodes are described (Scheme 1).

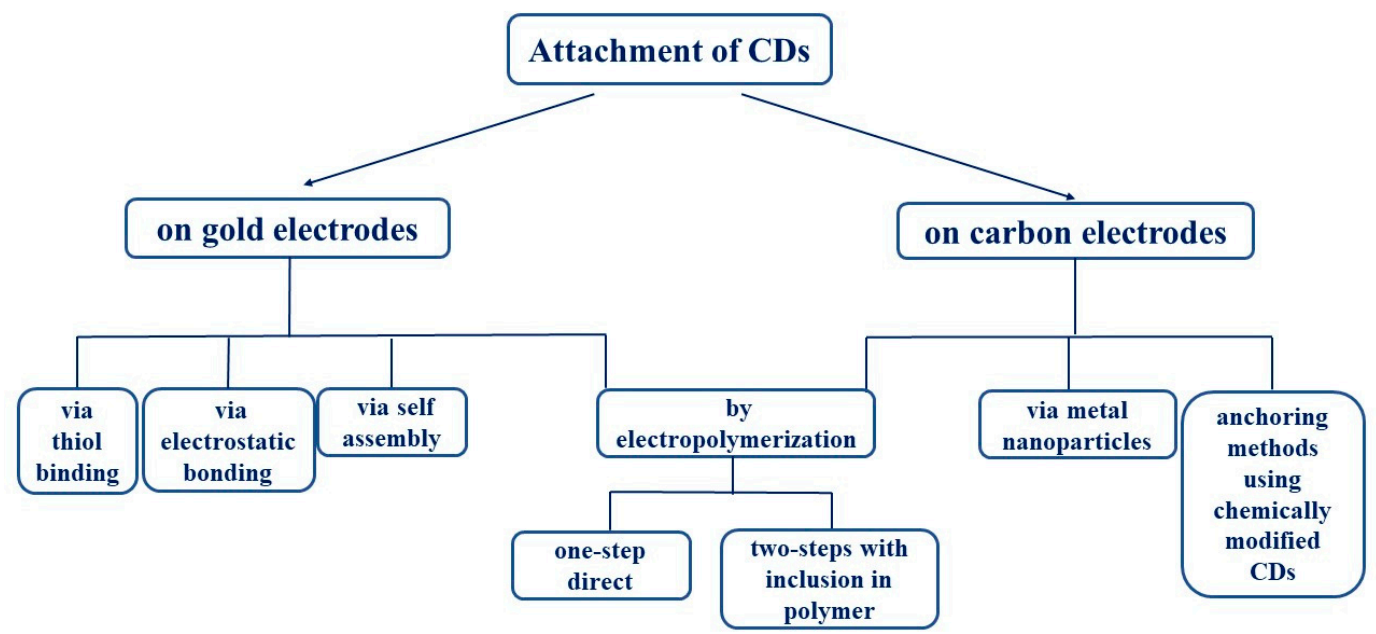

Scheme 1. Different methods of cyclodextrins (CDs) integration onto gold or carbon electrodes. 


\subsection{Inclusion of CDs onto Gold Electrode}

\subsubsection{Covalent Bond with $\mathrm{SH}-\beta-\mathrm{CDs}$}

Like previously reported, different $\mathrm{SH}-\beta$-CDs for electrochemical analysis have been considered in the last 30 years. They can be split in two main categories: "pure" SH- $\beta$-CDs (mono or multi-thiolated), and "not pure" SH- $\beta$-CDs, with the insertion of amino groups in the chain.

Dealing with the "pure" SH- $\beta$-CDs, Rojas et al. [10] synthetized multi-thiolated CDs derivatives that have been largely used in the following years to obtain CDs covalently bonded to gold electrodes or to GNPs. For example, Di Palma et al. [27] proposed a simple and reversible protein assemblies on well-oriented $\beta$-CD moieties tethered onto a gold surface.

In order to guarantee more mobility to the CDs once at the surface, and to facilitate the inclusion with target molecules [28], mono-thiolated CDs have been developed too. Fu et al. [9] synthetized mono-6-thio- $\beta$-CDs using the method of Wang et al. [11]. After the electrodeposition of gold nanoparticles (GNPs) onto carbon electrode previously functionalized with single-walled carbon nanotubes (SWCNTs), the electrode has been immersed in SH- $\beta$-CDs methanol solution to obtain an $\mathrm{Au}-\mathrm{SH}$ linkage. The pesticide methyl parathion has been then sensed by exploiting the host-guest inclusion property of the $\mathrm{SH}-\beta$-CDs.

\subsubsection{Electrostatic Bonding}

Even if the covalent binding of the thiolated group ensures the right orientation of CDs on the electrode surface, defect densities due to the lack of favorable lateral interaction between the CD molecules could occur [10]. Moreover, the incorporation of multiple and symmetrically distributed thiol groups on the CD could result in poor packing of the monolayer [26]. For these reasons, other approaches to obtain a well-oriented self-assembled-monolayer (SAM) of CDs onto the gold surface have been exploited.

One of these could be the interaction of CDs with gold electrode through opposite charges. Godinez et al. [29] demonstrated that the aggregation to the CDs of amphiphilic chains with positively charged amino groups not only permits a certain degree of packing and thickness of the multilayer assembly but also the specific orientation of the adsorbed amphiphiles.

\subsubsection{Self-Assembly}

The functionalization of CDs with long alkyl thiothers as spacers allows obtaining self-assembled structures with improved surface coverage and order [30]. However, the incorporation of alkyl chains decreases the conductivity of the organic molecules that hampers their use in applications based on electron transfer processes [31]. Therefore, alternatives to immobilize CDs with a proper orientation on gold electrodes without the synthesis of thiol-modified cyclodextrins is also proposed by bottom up techniques. These techniques typically consist on chemical modifications of gold surfaces in order to then subsequently bind CDs on top.

M. Garcia et al. [26] evaluated the possibility of immobilization of amino- $\beta$-CDs on gold surfaces via three different bottom up approaches: (i) direct adsorption, (ii) amide-bond formation with the carboxylic groups of a self-assembled monolayer of 4-mercapto benzoic acid (4-MBA) and (iii) covalent bond among carboxylic and amine groups of electrografted diazonium salt previously synthesized in situ by 4 -amino benzoic acid (4-ABA; Scheme 2$)$. 
A

$\mathrm{Au}$
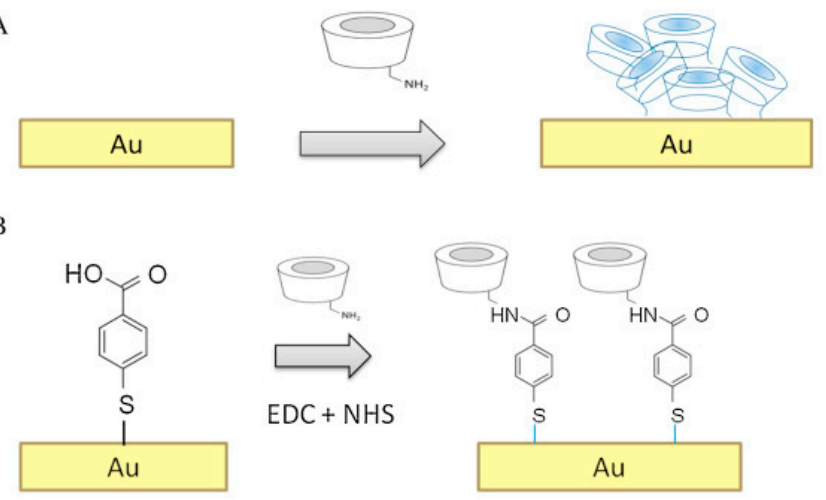

$\mathrm{C}$

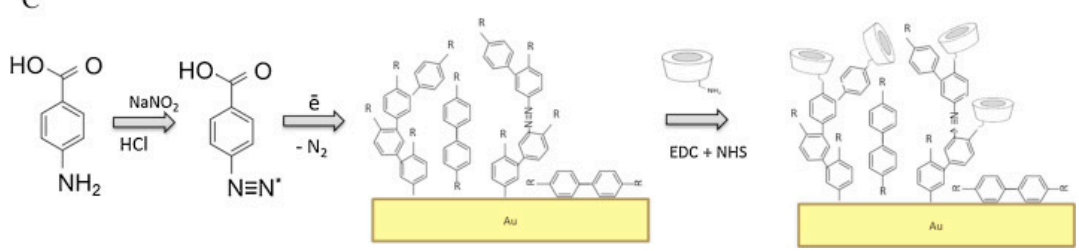

Scheme 2. Scheme about surface modification methods: (A) Direct adsorption of amino- $\beta$-CD onto gold surfaces; (B) covalent attachment by amide bond formation with carboxylic groups of a self assembled monolayer by 4-mercapto-benzoic acid. EDC is for N-(3-dimethylaminopropyl)-N0-ethylcarbodimide and NHS is for N-hydroxysulfosuccinimide; C) covalent bond between amine and carboxyl groups of the electrografted diazonium salt from 4-amino benzoic acid. Reproduced from [26], with permission from Elsevier, 2016.

The work of M. Garcia et al. demonstrated that, in the case of the well-ordered and packed thiolated layer, the surface coverage was proportional to the number of amine groups, while the porous structure of the electrografted diazonium layer allowed a higher level of surface coverage of disordered molecules. Then, Mèndez-Torres et al. [32] demonstrated the covalent immobilization of CDs to gold electrode by using the Garcia's bottom up strategy, but they obtained the CD cavity opposite oriented with respect to the gold surface. In such a configuration, the host-guest interactions can be enhanced since the CDs cavity is well exposed out of the surface. Their results show the feasibility to use such modified surfaces for molecular recognition as an alternative way to other more usually proposed conventional molecules, such as antigens, antibodies, enzymes, etc.

Yang et al. [33] proposed a different functionalization of three gold working electrodes (WEs) forming a multi-panel system for the detection of the three major catecholamine hormones: L-tyrosine, dihydroxyphenylalanine (L-DOPA) and dopamine. First, each one of the WEs was chemically treated to obtain a hydrophilic character. Then, each of the three WEs was finally functionalized with a different kind of CDs: different $\alpha-, \beta$ - or $\gamma$-CDs have been used in order to obtain sensors to selectively detect different hormones by exploiting the different size of the cavity (Scheme 3). 


\section{Surface functionalization}

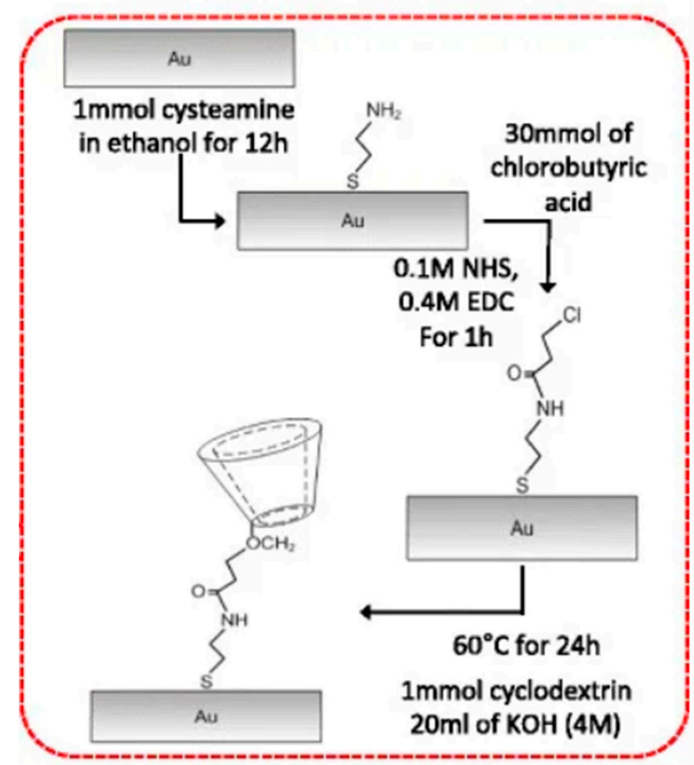

Scheme 3. Scheme of the surface functionalization of gold electrode with CDs by the bottom up method. Reproduced from [33], with permission from SpringerOpen, 2014.

It worth to note that, in this kind of bottom up approach, it is not required to use amino-CDs, while CDs of any other class of derivatives are possible to be used to functionalize the gold electrodes.

\subsection{Deposition Method for Both Gold and Carbon Electrode: Electropolymerization}

As an alternative to chemically modified CDs immobilization, electropolymerization of CDs has been successfully proposed too for the deposition of CDs onto sensing surfaces. There are different techniques of electropolymerization, mainly divided in two categories: (i) direct electropolymerization of CDs (direct electrodeposition of CDs), and (ii) inclusion (or entrapment) of CDs into electrodeposited polymeric films.

\subsubsection{Direct Electropolymerization of CDs}

This kind of polymerization could be considered as "one step" polymerization since it uses the property of CDs themselves to polymerize, while a "two-steps" electropolymerization is described in the Section 3.2.2.

Li et al. [34] developed an immunosensor by a simple electrochemical polymerization method (Scheme 4). A $\beta$-CD polymer (P $\beta-C D$ ) was used to modify the glassy carbon electrode (GCE). To enhance the electron transfer property of the modified electrode, and to facilitate the immobilization of the prion $\mathrm{Ab}$, gold nanoparticles (GNPs) were electrochemically deposited on the GCE surface and used as binding-bridge between $\mathrm{P} \beta-\mathrm{CD}$ film and GCE electrode. Bovine serum albumin (BSA) has been added in order to block the not-specific binding sites, a typical blocking strategy usually proposed to improve the selectivity of the sensing surface [35]. Used as a probe molecule, the ferrocyanide $\left(\mathrm{Fe}(\mathrm{CN})_{6}\right)$ provides electron transfer, and therefore a Faradaic current, even in the presence of CDs. While the cavity of CDs is blocked to the ferrocyanide in the presence of the prion antibody $(\operatorname{PrP})$ previously interacting to the respective antibody inserted in CDs channel. 


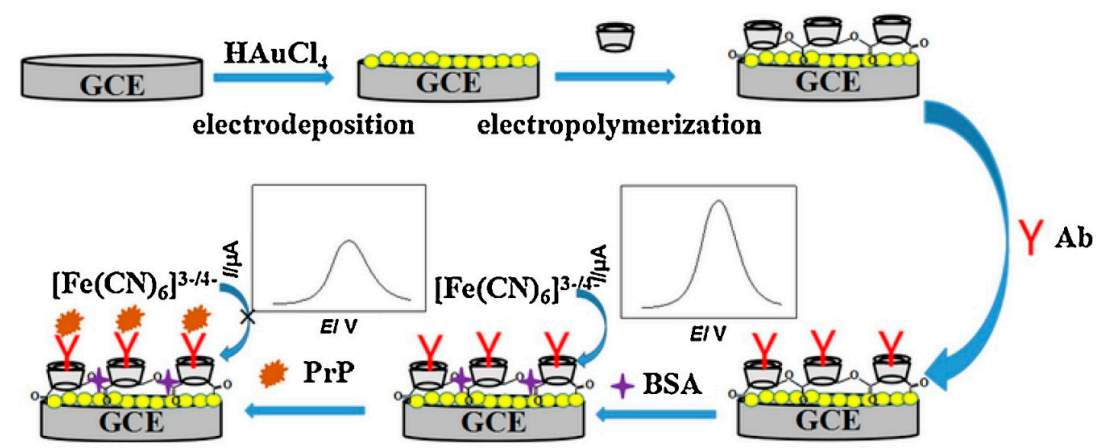

Scheme 4. Illustration about the fabrication procedure of electrochemical prion immunosensors. Glassy Carbon Electrode (GCE), Bovine Serum Albumin (BSA), prion antibody (PrP), antibody (Ab). Reproduced from [34], with permission from Elsevier, 2014.

Hui et al. [36] used carboxymethyl- $\beta$-CDs [6] as a dispersive agent for GNPs that have been electrodeposited onto carbon electrode. A first cyclic voltammetry with a $\mathrm{HAuCl}_{4} / \mathrm{CM}-\mathrm{CDs} \mathrm{PBS}$ solution leads to the formation of GNPs onto the electrode surface and a simultaneous functionalization with CM-CDs. Then, a second cyclic voltammetry with only CM-CDs PBS solution allowed the electropolymerization of CDs onto the GNPs surface. The sensor showed electrocatalysis to different drugs and other metabolites (e.g., the glucose) without the use of any specific enzyme (Scheme 5).

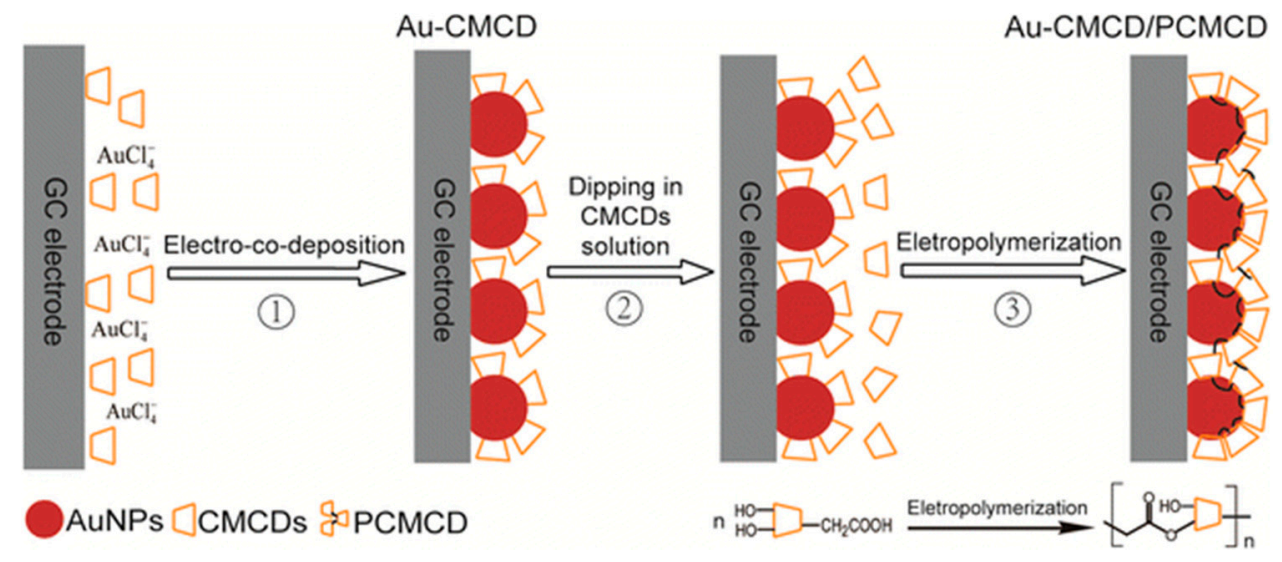

Scheme 5. Scheme of the fabrication of gold-carboxylmethyl-cyclodextrins (Au-CMCD) electrode and gold-carboxylmethyl-cyclodextrin/cyclodextrin-polymer (Au-CMCD/PCMCD) electrode. Reproduced from [36], with permission from Springer, 2016.

\subsubsection{Inclusion of CDs into a Polymer Film}

A common functionalization method is through two-step electropolymerization: first, a conductive polymer is electrodeposited onto electrode surface, and then CDs are added.

D'Souza et al. [37] developed a dioxygen sensor, by using the polycondensation of $\beta$-cyclodextrin soluble prepolymer ( $\beta$-CDPS) or of carboxymethylated $\beta$-cyclodextrin prepolymer ( $\beta$-CDPA), both hosting electrocatalytically active centers of cobalt tetraphenylporphyrin (TPP). The above-mentioned pre-polymers have been used as coating for the gold electrode surface.

Metal-organic framework (MOF) material with regular and orderly structure has attracted extensive attention for preparing sensors [38,39] as well. More recently, Wu et al. [40] developed a sensor with specific recognition cavities in imprinted microporous-metal-organic-framework (MI-MOF) membrane. Golden nanoparticles (GNPs) have been functionalized by SH- $\beta$-CDs and L-cysteine (L-Cys) and then electrodeposited onto electrode together with L-phenylalanine (L-Phe). Afterward, L-Phe has been removed from the CDs cavities by washing in methanol and acetic acid. Finally, 
cyclic voltammetry and differential pulse voltammetry measurements with different L-Phe target concentrations have demonstrated good selectivity respect to typical interfering molecules of the D-Phenylalanine (D-Phe; Scheme 6).

a

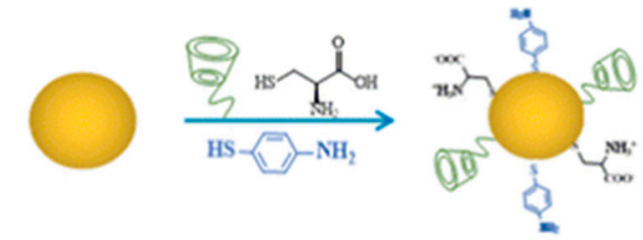

b
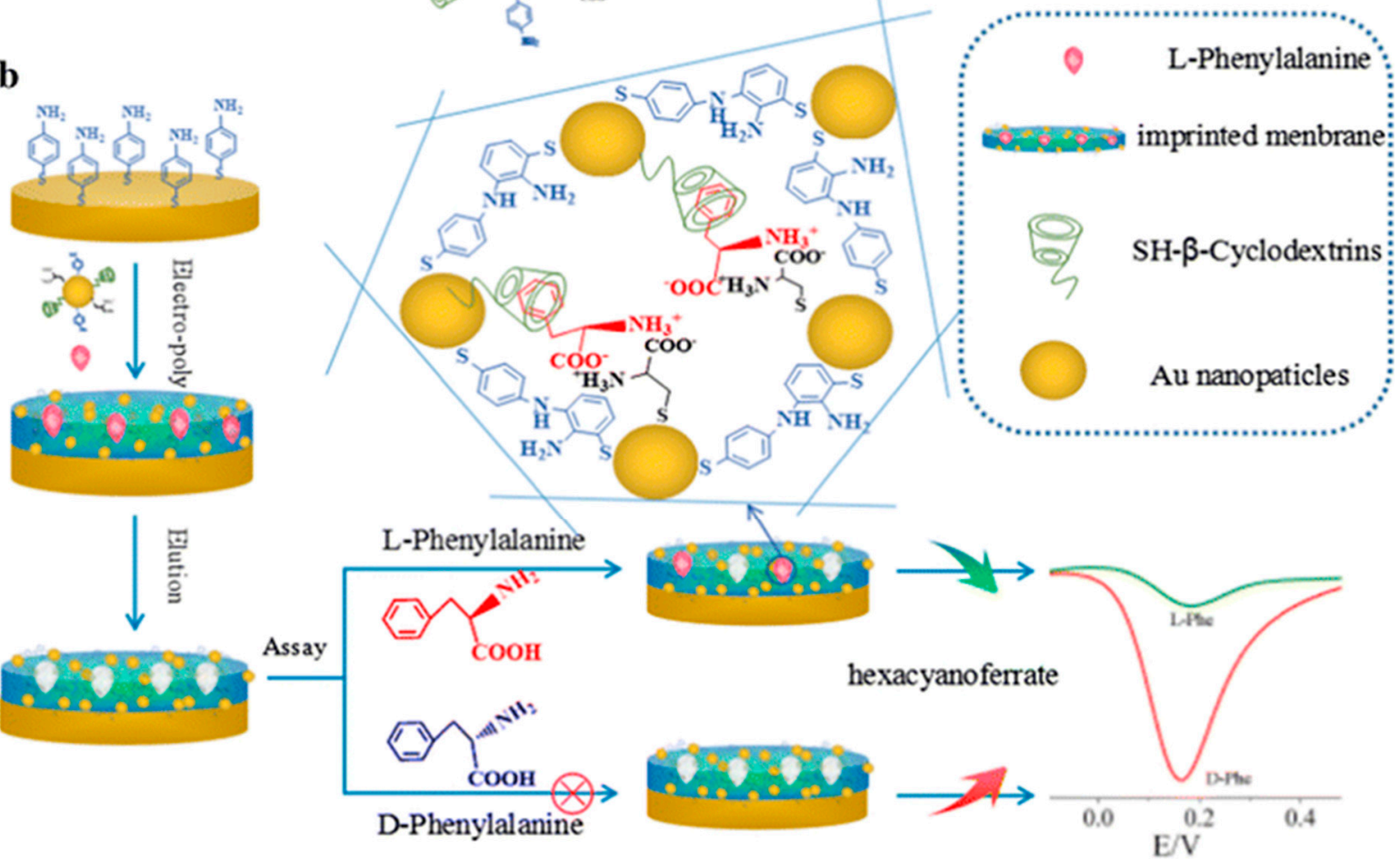

Scheme 6. Schematic of the miroporous metal-organic framework (MI-MOF) sensor. (a) Functionalization of Au nanoparticle with thiolated-CDs and L-Cysteine. (b) Electrodeposition of functionalized Au-nanoparticles and L-Phenylalanine on gold electrode. Reproduced from [40], with permission from Springer, 2017.

In this case, the selectivity is obtained by double recognition of CDs and L-cysteine. In fact, the hydrophobic benzene of L-phenylalanine (L-Phe) can be embedded in the CDs cavity, while the other end of the target molecule was captured by the L-cysteine through complementary electrical interactions by dipolar ions. However, D-phenylalanine (D-Phe) cannot be effectively recognized by imprinted cavities contributing to mismatch spatial configuration [40].

Arjomandi et al. [41,42] drugged N-methylpyrrole (NMPy) films with 2,6-dimethyl- $\beta$-cyclodextrin for electropolymerization onto gold electrode. G. Bidan et al. [43] did similar analysis for sulphonated cyclodextrins. Finally, Dermody et al. [44] deposited hyperbranched poly(acrylic acid) (PAA) films on Au electrodes. PAA film is a highly functionalized framework to covalently bind natural $\beta$-CDs, without any kind of their specific chemical modification.

CDs inclusion into a polymer film is also possible for the functionalization of carbon-based electrodes. For example, polyaniline (PANI) polymer is proposed for directly electrodeposition onto electrode surface [45] or onto a layer of CNTs [46], also in combination with L-glutanic acid (L-Glu) and CDs [47]. Co-electrodeposition of CDs and mono-dispersed nanocarbon onto glass carbon electrode (GCE) by $\beta-\mathrm{CD} /$ reduced-Graphene-Oxide $(\beta-\mathrm{CD} / \mathrm{rGO}$ ) has been proposed too by Jiang et al. [48].

Of course, a co-electropolymerization of $\mathrm{CDs}$ and another monomer-based solution is possible also avoiding the integration of carbon nanomaterials (CNTs or graphene). For example, Zhang et 
al. [49] developed a sensor for the detection of fluoroquinolones based on hydrogen interactions among L-arginine amino acid (L-arg) with CDs, while Bouchta et al. [50] achieved an electrosynthesis of 3-methylthiphene (P3MT) with $\beta$-CDs, through both the potentiostatic and galvanostatic method. Sun's group [51] polymerized mesoporous carbon onto GCE and, then, $\beta$-CDs were included into a poly-dopamine film for the detection of chloramphenicol (CAP).

\subsection{Inclusion of CDs onto Carbon Electrode}

In recent years, CDs-functionalized carbon materials (carbon nanotubes (CNTs), graphene and fullerene) [52] have been also developed and used in electrochemical sensors. In these cases, it is also possible to observe different functionalization techniques, like the simple mixing of carbon and CDs suspensions with successive drop-casting onto electrode surfaces $[53,54]$. Electropolymerization methods or chemical modifications of CDs on electrode surfaces have been employed too.

\subsubsection{Load of Metal Nanoparticles on Carbon-Based/ $\beta$-CDs Composites}

The host-guest inclusion typical property of CDs could be combined with the increase of active sites and surface area through the loading of metal nanoparticles on carbon-based/ $\beta$-CDs composite. $[5,55,56]$. For example, the surface modification of graphene with $\beta$-CDs through GNPs insertion could be a promising strategy for electrochemical enhanced sensing [57]. Taking advantage by thiol-binding due to the $\mathrm{SH}-\beta-\mathrm{CDs}$, carbon microspheres are functionalized with CDs due to the introduction of GNPs into carbon microsphere surface [58].

\subsubsection{Anchoring Methods Using Chemically Modified CDs}

In order to form covalent bonds directly with carbon substrates, CD chemical modifications are necessary.

For example, the carboxymethyl-CDs (CM-CDs)-based composites formed by covalent bonds exhibit high stability, compared to simple mixing, since CM-CDs present carboxyl groups necessary for amidation reaction. Chen et al. [59] used CM-CDs for functionalizing magnetic nanoparticles to design a sandwich immunoassay for the detection of ractopamine (RAC). On the other hand, if the carbon substrate is chemically modified with the $\mathrm{COOH}$ group, then amino-CDs can covalently bind. For instance, single-walled carbon nanotubes (SWCNTs) functionalized with 3,4,9,10-perylene tetracarboxylic acid (PTCA) and then stirred with $\mathrm{NH}_{2}-\beta-\mathrm{CD}$ based solution can bind CDs [60]. In a similar way, graphene could be also functionalized with $\mathrm{NH}_{2}-\mathrm{CDs}$ [23].

The HP-CDs derivatives have instead been used to improve the conductivity of carbon electrode for the detection of glutathione (GSH) by using 10-methylphenothiozine (MPT). In fact, the MPT molecules can enter into CDs cavities. In this way, MPT could more rapidly exchange electrons with the electrode and perform the catalytic oxygen reduction of GSH [16]. In many other cases, HP-CDs are immobilized onto graphene [61] or graphene nanoribbons [62] and then the complex is cast on the carbon electrode surface.

We mentioned already the SH group as being fundamental for the CDs covalent binding with gold substrates, but there is one case where it would be possible to use this kind of CD derivatives to directly attach on the carbon substrate. This was done by Yang et al. [63] through the synthesis of a kind of bridged-bis-( $\beta-C D s)$, starting from a pair of $\mathrm{SH}-\beta-\mathrm{CDs}$. It seems that two adjacent $\mathrm{CDs}$ units exhibit more significantly binding abilities and molecular recognition than native or mono-modified CDs. The disulfides bridged $\beta$-CD have been bonded to MWCNTs and then dropped onto the sensor for the detection of three different kinds of phenol.

Finally, another interesting and not so-commonly-used CDs chemical modification is the introduction of the pyrene group. In fact, a strong hydrophobic-hydrophobic interaction is guaranteed among CDs and carbon material due to this chemical group, even if this is not a covalent bond. Moreover, CDs result was well exposed and right orientated to the exterior due to the presence of a certain chain. In literature, they have been bonded to CNTs [64,65] (Scheme 7) but they looked 
at the only kind of CDs that could be bonded directly to glass carbon electrode (GCE) through an immersion of the electrodes in pyrene-CDs solution, without the need of any sonication, centrifugation or filtration.

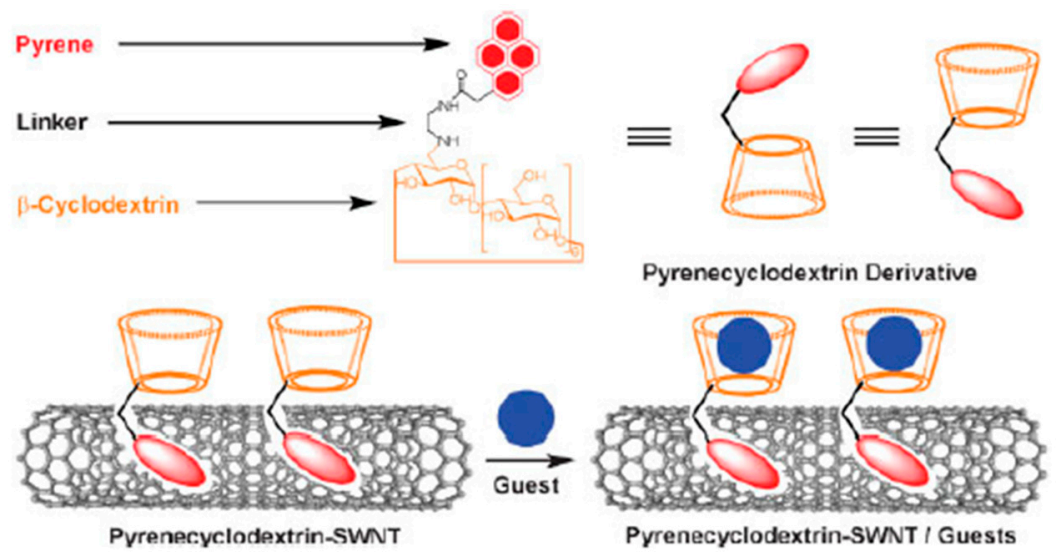

Scheme 7. Schematic drawing of pyrenecyclodextrin-decorated single-walled carbon nanotube (SWCNT) hybrids and their interaction with target molecules. Reproduced from [64], with permission from John Wiley and Sons, 2008.

\section{CDs for Biomedical Devices}

Many of the electrodes functionalizations with CDs examined in this review article have been fabricated for the detection of molecules related to biomedical detection.

In the most of the cases, the target molecule possesses hydrophobic parts that allow the match with CDs hydrophobic cavities and to facilitate their absorption onto electrode surface. In this case, direct detection is achieved due to the fact that the target molecule is electroactive, so CDs are used as a selective bridge in order to help the hydrophobic target to reach the electrode surface and to directly exchange electrons with the electrodes $[33,40,66]$.

Other types of bio-probes can be: not electroactive, which binds with the target molecule, while the redox reactions take place by other molecules $[34,67,68]$; while electroactive probes like hydroquinone [45] or ferrocene [69,70] are used in order to detect all those biomolecules that do not possess any electroactive sites. This last kind of detection is indirect detection typically based on competition of electroactive probes and target molecules on CDs. The electroactive probe could enter in the CD cavity instead of the target, increasing its exchanging of electrons with the electrode, which results in a decrease of current (Scheme 8). 


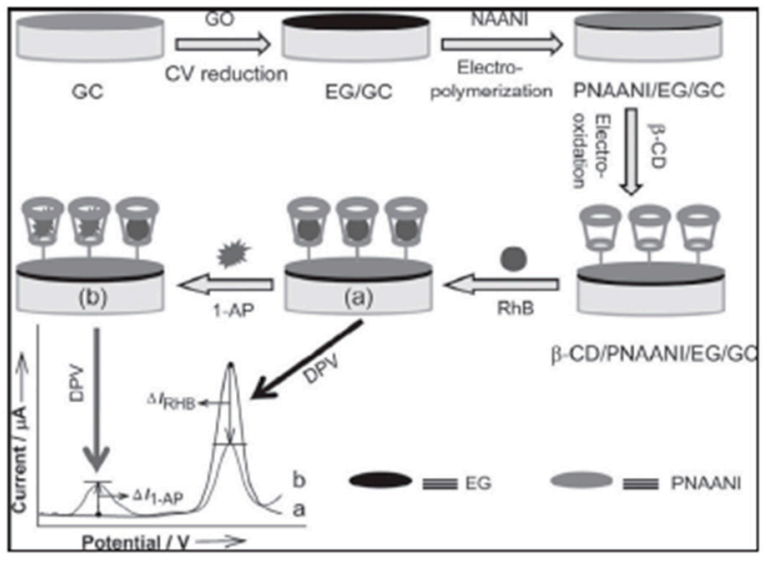

Scheme 8. Assay protocol of dual-signaling electrochemical sensor based on the competitive host-guest interaction between $\beta-C D$ and rhodamine $B(\mathrm{RhB})$ (reporting probe)/1-aminopyrene (1-AP) (target). GC is for Glass Carbon (electrode), GO is for Graphene Oxide, CV is for Cyclic Voltammetry, EG/GC is for Graphene/Carbon (electrode), NAANI is for N-acetylaniline, DPV is for Double Pulse Voltammetry. Reproduced from [71], with permission from John Wiley and Sons, 2013.

The reduced size of CDs permits the detection of many biomolecules whose sizes are inferior of 12-15 nm, like drugs, amino acids, etc. while many other targets may have larger sizes. In this case, CDs are used simply as a dispersing agent of nanoparticles (e.g., of golden nanoparticles (GNPs)) that may better dissolve, reduce their diameter and make the effective surface area increase for enzyme-less detection of glucose [36]. Moreover, Hishiya et al. [72] used CDs immobilized into a conductive polymer as a template to bind hydrophobic parts of cholesterol as shown in Scheme 9.

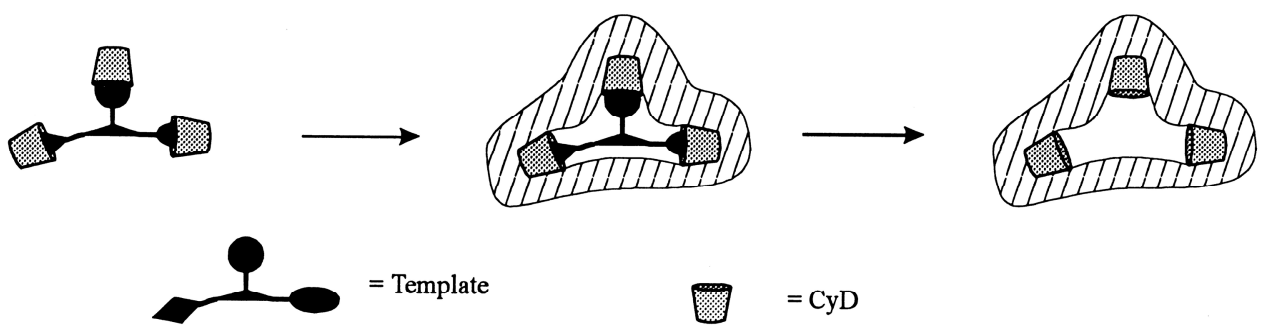

Scheme 9. Molecular imprinting of CD to obtain assemblies as tailor-made receptors. Reproduced from [72], with permission from American Chemical Society, 1999.

\section{Conclusions}

We described in this paper the deposition of CDs for electrochemical sensing. We considered here many CDs chemical modifications, starting from their typical "host-guest complex" shape created by their toroidal structure and then considering different options to further functionalized the molecules for deposition aims. Chemical modifications typically are required to improve their binding properties, their solubility and especially to achieve a better incorporation onto the sensing electrodes.

We focused our attention particularly on different techniques of CDs inclusion onto gold and carbon electrodes. Some techniques consider chemical modifications of CDs (thiolation or pyrene-CDs), others chemical modification of the electrodes (bottom up); or CDs direct electropolymerization (direct electropolymerization) or inclusion of CDs into other conductive polymers then further deposited by electropolymerization.

CDs use in the detection of metabolites and other biomolecules is becoming more relevant in literature for the detection of small organic molecules. The recognition of molecules such as glucose or cholesterol has been already demonstrated. In this respect, CDs have a huge potential as recognition elements once incorporated on electrodes, in order to provide the right selectivity to the final sensing 
device. The chemical modifications of CDs surfaces open the possibility to explore a variety of sensing mechanisms for several new applications to diagnostics, life science and biology.

Author Contributions: Original draft preparation and writing, M.A.C.; methodology, I.T.; supervision, S.C. and T.H.

Funding: This research received no external funding.

Acknowledgments: Generous financial support from Sophia Alumni Entrepreneurs Club Scholarship, Sophia University are gratefully acknowledged.

Conflicts of Interest: The authors declare no conflict of interest.

\section{References}

1. Boero, C.; Casulli, M.A.; Olivo, J.; Foglia, L.; Orso, E.; Mazza, M.; Carrara, S.; De Micheli, G. Design, development, and validation of an in-situ biosensor array for metabolite monitoring of cell cultures. Biosens. Bioelectron. 2014, 61, 251-259. [CrossRef]

2. Baj-Rossi, C.; De Micheli, G.; Carrara, S. Electrochemical detection of anti-breast-cancer agents in human serum by cytochrome P450-coated carbon nanotubes. Sensors 2012, 12, 6520-6537. [CrossRef]

3. Park, S.; Boo, H.; Dong, T. Electrochemical non-enzymatic glucose sensors. Anal. Chim. Acta 2006, 556, 46-57. [CrossRef] [PubMed]

4. Baj-Rossi, C.; Rezzonico Jost, T.; Cavallini, A.; Grassi, F.; De Micheli, G.; Carrara, S. Continuous monitoring of Naproxen by a cytochrome P450-based electrochemical sensor. Biosens. Bioelectron. 2014, 53, 283-287. [CrossRef] [PubMed]

5. Niu, X.; Mo, Z.; Yang, X.; Sun, M.; Zhao, P.; Li, Z.; Ouyang, M.; Liu, Z.; Gao, H.; Guo, R.; et al. Advances in the use of functional composites of $\beta$-cyclodextrin in electrochemical sensors. Microchim. Acta 2018, 185, 328. [CrossRef] [PubMed]

6. Badruddoza, A.Z.M.; Si, G.; Hazel, S.; Hidajat, K.; Uddin, M.S. Synthesis of carboxymethyl- $\beta$-cyclodextrin conjugated magnetic nano-adsorbent for removal of methylene blue. Colloids Surfaces A Physicochem. Eng. Asp. 2010, 367, 85-95. [CrossRef]

7. Badruddoza, A.Z.M.; Tay, A.S.H.; Tan, P.Y.; Hidajat, K.; Uddin, M.S. Carboxymethyl- $\beta$-cyclodextrin conjugated magnetic nanoparticles as nano-adsorbents for removal of copper ions: Synthesis and adsorption studies. J. Hazard. Mater. 2011, 185, 1177-1186. [CrossRef]

8. Wu, S.; Fan, S.; Tan, S.; Wang, J.; Li, C. A new strategy for the sensitive electrochemical determination of nitrophenol isomers using $\beta$-cyclodextrin derivative-functionalized silicon carbide. RSC Adv. 2018, 8, 775-784. [CrossRef]

9. $\mathrm{Fu}, \mathrm{X}$; Zhang, J.; Tao, Y.; Wu, J.; Xie, C.; Kong, L. Three-dimensional mono-6-thio- $\beta$-cyclodextrin covalently functionalized gold nanoparticle/single-wall carbon nanotube hybrids for highly sensitive and selective electrochemical determination of methyl parathion. Electrochim. Acta 2015, 153, 12-18. [CrossRef]

10. Rojas, M.T.; Konigety, R.; Stoddart, J.F.; Kaifer, A.E. Supported monolayers containing preformed binding sites. synthesis and interfacial binding properties of a thiolated/3-cyclodextrin derivative. J. Am. Chem. Soc. 1995, 117, 336-343. [CrossRef]

11. Wang, J.; Kong, L.; Guo, Z.; Liu, J. Synthesis of novel decorated one-dimensional gold nanoparticle and its application in ultrasensitive detection of insecticide. J. Mater. Chem. 2010, 20, 5271-5279. [CrossRef]

12. Weisser, M.; Nelles, G.; Wohlfart, P.; Wenz, G.; Mittler-Neher, S. Immobilization kinetics of cyclodextrins at gold surfaces. J. Phys. Chem. 1996, 3654, 17893-17900. [CrossRef]

13. Maeda, Y.; Fukuda, T.; Yamamoto, H.; Kitano, H. Regio-and stereoselective complexation by a self-assembled monolayer of thiolated cyclodextrin on a gold electrode. Langmuir 1997, 13, 4187-4189. [CrossRef]

14. Kitano, H.; Taira, Y.; Yamamoto, H. Inclusion of phthalate esters by a self-assembled monolayer of thiolated cyclodextrin on a gold electrode. Anal. Chem. 2000, 72, 2976-2980. [CrossRef]

15. Maeda, Y.; Kitano, H. Inclusional complexation by cyclodextrins at the surface of silver as evidenced by surface-enhanced resonance Raman spectroscopy. J. Phys. Chem. 1995, 99, 487-488. [CrossRef]

16. Li, X.; Zheng, L.; Wang, Y.; Zhang, N.; Lou, Y.; Xiao, T.; Liu, J. A novel electrocatalyst with high sensitivity in detecting glutathione reduced by 2 -hydroxypropyl- $\beta$-cyclodextrin enveloped 10-methylphenothiazine. RSC Adv. 2015, 5, 71749-71755. [CrossRef] 
17. Ye, L.; Huang, N.; Du, Y.; Schneider, M.; Du, W. Succinyl- $\beta$-cyclodextrin modified gold biochip improved seroimmunological detection sensitivity for Lyme disease. Anal. Chim. Acta 2017, 953, 48-56. [CrossRef]

18. Zhou, Y.; Zhao, J.; Li, S.; Guo, M.; Fan, Z. An electrochemical sensor for the detection of: P -nitrophenol based on a cyclodextrin-decorated gold nanoparticle-mesoporous carbon hybrid. Analyst 2019, 144, 4400-4406. [CrossRef]

19. Hea, P.; Fanga, Y.; Suzukib, I.; Osab, T. Voltammetric responsive sensors for organic compounds based on organized self-assembled lipoyl-P-cyclodextrin derivative monolayer on a gold electrode. Anal. Chim. Acta 1997, 337, 217-223. [CrossRef]

20. Tang, B.; Liang, H.; Xu, K.; Mao, Z.; Shi, X.; Chen, Z. An improved synthesis of disulfides linked $\beta$-cyclodextrin dimer and its analytical application for dequalinium chloride determination by spectrofluorimetry. Anal. Chim. Acta 2005, 554, 31-36. [CrossRef]

21. Liu, J.; Ong, W.; Román, E.; Lynn, M.J.; Kaifer, A.E. Cyclodextrin-modified gold nanospheres. Langmuir 2000, 16, 3000-3002. [CrossRef]

22. Kong, L.; Wang, J.; Meng, F.; Chen, X.; Jin, Z.; Li, M.; Liu, J.; Huang, X.J. Novel hybridized SWCNT-PCD: Synthesis and host-guest inclusion for electrical sensing recognition of persistent organic pollutants. J. Mater. Chem. 2011, 21, 11109-11115. [CrossRef]

23. Tan, L.; Wang, G.; Chen, N.; Zhang, J.; Feng, H. Layer-by-layer assembled multilayers of graphene/mono-(6-amino-6-deoxy)- $\beta$-cyclodextrin for detection of dopamine. Chin. J. Chem. 2015, 33, 185-191. [CrossRef]

24. Zhang, Q.; Bai, Z.; Shi, M.; Yang, L.; Qiao, J.; Jiang, K. High-efficiency palladium nanoparticles supported on hydroxypropyl- $\beta$-cyclodextrin modified fullerene [60] for ethanol oxidation. Electrochim. Acta 2015, 177, 113-117. [CrossRef]

25. Shang, F.; Zhou, L.; Mahmoud, K.A.; Hrapovic, S.; Liu, Y.; Moynihan, H.A.; Glennon, J.D.; Luong, J.H. Selective nanomolar detection of dopamine using a boron-doped diamond electrode modified with an electropolymerized sulfobutylether- $\beta$-cyclodextrin-doped poly (N-acetyltyramine) and polypyrrole composite film. Anal. Chem. 2009, 81, 4089-4098. [CrossRef]

26. García, M.; Bollo, S.; Rivas, G.A.; Ferreyra, N.F.; Yáñez, C. Bottom up approaches for amino $\beta$-CD adsorption on gold surfaces. A comparative study. Electrochim. Acta 2016, 203, 292-300. [CrossRef]

27. Di Palma, G.; Kotowska, A.M.; Hart, L.R.; Scurr, D.J.; Rawson, F.J.; Tommasone, S.; Mendes, P.M. Reversible, high-affinity surface capturing of proteins directed by supramolecular assembly. ACS Appl. Mater. Interfaces 2019, 11, 8937-8944. [CrossRef]

28. Labuda, A.F.J. Cyclodextrins as electrode modifiers. Fresenius' J. Anal. Chem. 2001, 370, 1-10.

29. Godínez, L.A.; Lin, J.; Muñoz, M.; Coleman, A.W.; Rubin, S.; Parikh, A.; Zawodzinski, T.A.; Loveday, D.; Ferraris, J.P.; Kaifer, A.E. Multilayer self-assembly of amphiphilic cyclodextrin hosts on bare and modified gold substrates: Controlling aggregation via surface modification. Langmuir 1998, 14, 137-144.

30. Veerbeek, J.; Méndez-Ardoy, A.; Huskens, J. Self-assembled monolayers of heptapodant $\beta$-cyclodextrins on gold. Langmuir 1998, 14, 6424-6429.

31. Steentjes, T.; Kudernac, T.; Huskens, J. Self-assembled monolayers on gold of $\beta$-cyclodextrin adsorbates with different anchoring groups. Langmuir 2014, 30, 3467-3476.

32. Méndez-Torres, A.M.; Sandoval-Altamirano, C.; Sánchez-Arenillas, M.; Marco, J.F.; Yáñez, C. Amino $\beta$-cyclodextrins immobilized on gold surfaces: Effect of substituents on host-guest interactions. Electrochim. Acta 2018, 282, 860-869.

33. Yang, J.; Kim, H.T.; Kim, H. A cyclodextrin-based approach for selective detection of catecholamine hormone mixtures. Micro Nano Syst. Lett. 2014, 2, 1-10. [CrossRef]

34. Li, X.; Li, J.; Liu, Y.; Zhang, X.; Chen, J. A sensitive electrochemical immunosensor for prion detection based on poly- $\beta$-cyclodextrin/gold nanoparticles/glassy carbon electrode. Sens. Actuators B Chem. 2017, 250, 1-7. [CrossRef]

35. Abdorahim, M.; Rabiee, M.; Alhosseini, S.N.; Tahriri, M.; Yazdanpanah, S.; Alavi, S.H.; Tayebi, L. Nanomaterials-based electrochemical immunosensors for cardiac troponin recognition: An illustrated review. Trends Anal. Chem. 2016, 82, 337-347. [CrossRef]

36. Hui, Y.; Ma, X.; Qu, F.; Chen, F.; Yu, J. Electropolymerization of carboxymethyl- $\beta$-cyclodextrin based on co-electrodeposition gold nanoparticles electrode: Electrocatalysis and nonenzymatic glucose sensing. J. Solid State Electrochem. 2016, 20, 1377-1389. [CrossRef] 
37. Souza, F.D.; Hsieh, Y.; Wickman, H.; Kutner, W. $\beta$-cyclodextrin and carboxymethylated $\beta$-cyclodextrin polymer film modified electrodes, hosting cobalt porphyrins, as sensors for electrocatalytic determination of oxygen dissolved in solution. Electroanalysis 1997, 9, 1093-1101. [CrossRef]

38. Guo, Z.; Florea, A.; Cristea, C.; Bessueille, F.; Vocanson, F.; Goutaland, F.; Zhang, A.; Săndulescu, R.; Lagarde, F.; Jaffrezic-Renault, N. 1, 3, 5-Trinitrotoluene detection by a molecularly imprinted polymer sensor based on electropolymerization of a microporous-metal-organic framework. Sens. Actuators B Chem. 2015, 207, 960-966. [CrossRef]

39. Florea, A.; Guo, Z.; Cristea, C.; Bessueille, F.; Vocanson, F.; Goutaland, F.; Dzyadevych, S.; Săndulescu, R.; Jaffrezic-Renault, N. Anticancer drug detection using a highly sensitive molecularly imprinted electrochemical sensor based on an electropolymerized microporous metal organic framework. Talanta 2015, 138, 71-76. [CrossRef]

40. Wu, T.; Wei, X.; Ma, X.; Li, J. Amperometric sensing of L-phenylalanine using a gold electrode modified with a metal organic framework, a molecularly imprinted polymer, and $\beta$-cyclodextrin-functionalized gold nanoparticles. Microchim. Acta 2017, 184, 2901-2907. [CrossRef]

41. Arjomandi, J.; Holze, R. Electrochemical preparation and in situ characterization of poly(3-methylpyrrole) and poly(3-methylpyrrole-cyclodextrin) films on gold electrodes. Open Chem. 2008, 6, 199-207. [CrossRef]

42. Arjomandi, J.; Holze, R. In situ characterization of N-methylpyrrole and (N-methylpyrrole-cyclodextrin) polymers on gold electrodes in aqueous and nonaqueous solution. Synth. Met. 2007, 157, 1021-1028. [CrossRef]

43. Bidan, G.; Lopez, C.; Vieil, E. Incorporation of suiphonated cyclodextrins into polypyrrole: An approach for the electro-controlled delivering of neutral drugs. Biosens. Bioelectron. 1994, 9, 219-229.

44. Dermody, D.L.; Peez, R.F.; Bergbreiter, D.E.; Crooks, R.M. Chemically grafted polymeric filters for chemical sensors: Hyperbranched poly (acrylic acid) films incorporating -cyclodextrin receptors and amine-functionalized filter layers. Langmuir 1999, 15, 885-890. [CrossRef]

45. Zheng, L.; Wu, S.; Lin, X.; Nie, L.; Rui, L. Preparation and characterization of a novel -cyclodextrin modified poly ( $\mathrm{N}$-acetylaniline) film. Macromolecules 2002, 6174-6177. [CrossRef]

46. Abbaspour, A.; Noori, A. A cyclodextrin host-guest recognition approach to an electrochemical sensor for simultaneous quantification of serotonin and dopamine. Biosens. Bioelectron. 2011, 26, 4674-4680. [CrossRef]

47. Tao, Y.; Dai, J.; Kong, Y.; Sha, Y. Temperature-sensitive electrochemical recognition of tryptophan enantiomers based on $\beta$-cyclodextrin self-assembled on poly(l-glutamic acid). Anal. Chem. 2014, 86, 2633-2639. [CrossRef]

48. Jiang, Z.; Li, G.; Zhang, M. Electrochemical sensor based on electro-polymerization of $\beta$-cyclodextrin and reduced-graphene oxide on glassy carbon electrode for determination of gatifloxacin. Sens. Actuators B Chem. 2016, 228, 59-65. [CrossRef]

49. Zhang, F.; Gu, S.; Ding, Y.; Zhang, Z.; Li, L. A novel sensor based on electropolymerization of $\beta$-cyclodextrin and 1-arginine on carbon paste electrode for determination of fluoroquinolones. Anal. Chim. Acta 2013, 770, 53-61. [CrossRef]

50. Bouchta, D.; Izaoumen, N.; Zejli, H.; El Kaoutit, M.; Temsamani, K.R. A novel electrochemical synthesis of poly-3-methylthiophene-gamma-cyclodextrin film. Application for the analysis of chlorpromazine and some neurotransmitters. Biosens. Bioelectron. 2005, 20, 2228-2235. [CrossRef]

51. Sun, Y.; Wei, T.; Jiang, M.; Xu, L.; Xu, Z. Voltammetric sensor for chloramphenicol determination based on a dual signal enhancement strategy with ordered mesoporous carbon@polydopamine and $\beta$-cyclodextrin. Sens. Actuators B Chem. 2018, 255, 2155-2162. [CrossRef]

52. Zhu, G.; Yi, Y.; Chen, J. Recent advances for cyclodextrin-based materials in electrochemical sensing. Trends Anal. Chem. 2016, 80, 232-241. [CrossRef]

53. Palanisamy, S.; Thirumalraj, B.; Chen, S. A novel amperometric nitrite sensor based on screen printed carbon electrode modified with graphite/ $\beta$-cyclodextrin composite. J. Electroanal. Chem. 2016, 760, 97-104. [CrossRef]

54. Zaidi, S.A. Facile and efficient electrochemical enantiomer recognition of phenylalanine using $\beta$-cyclodextrin immobilized on reduced graphene oxide. Biosens. Bioelectron. 2017, 94, 714-718. [CrossRef] [PubMed]

55. Kushikawa, R.T.; Silva, M.R.; Angelo, A.C.D.; Teixeira, M.F.S. Construction of an electrochemical sensing platform based on platinum nanoparticles supported on carbon for tetracycline determination. Sens. Actuators B Chem. 2016, 228, 207-213. [CrossRef] 
56. Palanisamy, S.; Kokulnathan, T.; Chen, S.; Velusamy, V.; Kannan, S. Voltammetric determination of Sudan I in food samples based on platinum nanoparticles decorated on graphene- $\beta$-cyclodextrin modified electrode. J. Electroanal. Chem. 2017, 794, 64-70. [CrossRef]

57. Tian, X.; Cheng, C.; Yuan, H.; Du, J.; Xiao, D.; Xie, S.; Choi, M.M. Simultaneous determination of L-ascorbic acid, dopamine and uric acid with gold nanoparticles- $\beta$-cyclodextrin-graphene-modified electrode by square wave voltammetry. Talanta 2012, 93, 79-85. [CrossRef]

58. Zhu, G.; Gai, P.; Yang, Y.; Zhang, X.; Chen, J. Electrochemical sensor for naphthols based on gold nanoparticles/hollow nitrogen-doped carbon microsphere hybrids functionalized with. Anal. Chim. Acta 2012, 723, 33-38. [CrossRef]

59. Chen, S.; Zhang, J.; Gan, N.; Hu, F. An on-site immunosensor for ractopamine based on a personal glucose meter and using magnetic $\beta$-cyclodextrin-coated nanoparticles for enrichment, and an invertase-labeled nanogold probe for signal amplification. Microchim. Acta 2015, 182, 815-822. [CrossRef]

60. Zhu, G.; Zhang, X.; Gai, P.; Zhang, X.; Chen, J. $\beta$-Cyclodextrin non-covalently functionalized single-walled carbon nanotubes bridged by 3, 4, 9, 10-perylene tetracarboxylic acid for ultrasensitive electrochemical sensing of 9-anthracenecarboxylic acid. Nanoscale 2012, 4, 5703-5709. [CrossRef]

61. Chem, J.M.; Xu, C.; Wang, J.; Wan, L.; Lin, J.; Wang, X. Microwave-assisted covalent modification of graphenenanosheets with hydroxypropyl- $\beta$-cyclodextrin and its electrochemical detection of phenolic organic pollutants. J. Mater. Chem. 2011, 21, 10463-10471.

62. Zhu, G.; Yi, Y.; Liu, Z.; Jin, H.; Chen, J. Electrochemistry communications highly sensitive electrochemical sensing based on 2-hydroxypropyl- $\beta$-cyclodextrin-functionalized graphene nanoribbons. Electrochem. Commun. 2016, 66, 10-15. [CrossRef]

63. Yang, L.; Fan, S.; Deng, G.; Li, Y.; Ran, X.; Zhao, H. Bridged $\beta$-cyclodextrin-functionalized MWCNT with higher supramo- lecular recognition capability: The simultaneous electrochemical determination of three phenols. Biosens. Bioelectron. 2015, 68, 617-625. [CrossRef] [PubMed]

64. Zhao, B.Y.; Hu, L.; Stoddart, J.F.; Gru, G. Pyrenecyclodextrin-decorated single-walled carbon nanotube field-effect transistors as chemical sensors. Adv. Mater. 2008, 20, 1910-1915. [CrossRef]

65. Wei, Y.; Kong, L.; Yang, R.; Wang, L.; Liu, J.; Huang, X. Single-walled carbon nanotube/pyrenecyclodextrin nanohybrids for ultrahighly sensitive and selective detection of $\mathrm{p}$-nitrophenol. Langmuir 2011, 27, 10295-10301. [CrossRef] [PubMed]

66. Yang, L.; Zhao, H.; Li, C.; Fan, S.; Li, B. Dual $\beta$-cyclodextrin functionalized Au@SiC nanohybrids for the electrochemical determination of tadalafil in the presence of acetonitrile. Biosens. Bioelectron. 2015, 64, 126-130. [CrossRef] [PubMed]

67. Cova, T.F.; Murtinho, D.; Pais, A.A.C.C.; Valente, A.J.M. Combining cellulose and cyclodextrins: Fascinating designs for materials and pharmaceutics. Front. Chem. 2018, 6, 1-19. [CrossRef]

68. Gao, J.; Guo, Z.; Su, F.; Gao, L.; Pang, X.; Cao, W.; Du, B.; Wei, Q. Ultrasensitive electrochemical immunoassay for CEA through host-guest interaction of $\beta$-cyclodextrin functionalized graphene and $\mathrm{Cu} @ \mathrm{Ag}$ core-shell nanoparticles with adamantine-modified antibody. Biosens. Bioelectron. 2015, 63, 465-471. [CrossRef]

69. Koradecki, D.; Kutner, W. Inclusion of the regioisomeric nitrobenzene derivatives and ferrocene guests by $\beta$-cyclodextrin polymer and their transport through the polymer matrix. J. Incl. Phenom. Mol. Recognit. Chem. 1991, 79-96. [CrossRef]

70. Ju, H. Host-guest interaction at a self-assembled monolayer/solution interface: An electrochemical analysis of the inclusion of 11-(Ferrocenylcarbonyloxy)undecanethiol by cyclodextrins. Langmuir 1998, 14, 300-306. [CrossRef]

71. Zhu, G.; Wu, L.; Zhang, X.; Liu, W.; Zhang, X.; Chen, J. A New dual-signalling electrochemical sensing strategy based on competitive host-guest interaction of a b-cyclodextrin/poly (N-acetylaniline)/graphene-modified electrode: Sensitive electrochemical determination of organic pollutants. Chem. Eur. J. 2013, 19, 6368-6373. [CrossRef] [PubMed]

72. Hishiya, T.; Shibata, M.; Kakazu, M.; Asanuma, H.; Komiyama, M. Molecularly imprinted cyclodextrins as selective receptors for steroids. Macromolecules 1999, 32, 2265-2269. [CrossRef]

(C) 2019 by the authors. Licensee MDPI, Basel, Switzerland. This article is an open access article distributed under the terms and conditions of the Creative Commons Attribution (CC BY) license (http://creativecommons.org/licenses/by/4.0/). 